https://helda.helsinki.fi

\title{
Inhomogeneous particle model for light-scattering by cometary dust
}

\section{Markkanen, Henri Johannes}

2015

Markkanen , H J , Penttilä , A I , Peltoniemi , J I \& Muinonen , K O 2015 , ' Inhomogeneous particle model for light-scattering by cometary dust ' , Planetary and Space Science, vol. 118 , pp. 164-172 . https://doi.org/10.1016/j.pss.2015.06.006

http://hdl.handle.net/10138/159454

https://doi.org/10.1016/j.pss.2015.06.006

acceptedVersion

Downloaded from Helda, University of Helsinki institutional repository.

This is an electronic reprint of the original article.

This reprint may differ from the original in pagination and typographic detail.

Please cite the original version. 


\title{
Inhomogeneous particle model for light-scattering by cometary dust
}

\author{
Johannes Markkanen,, Antti Penttiläa ${ }^{\mathrm{a}, \text { Jouni Peltoniemi }}{ }^{\mathrm{a}, \mathrm{b}}$, Karri Muinonen ${ }^{\mathrm{a}, \mathrm{b}}$ \\ ${ }^{a}$ Department of Physics, PO Box 64, FI-00014, University of Helsinki, Finland \\ ${ }^{b}$ Finnish Geospatial Research Institute FGI, Geodeentinrinne 2, FI-02430, Masala, Finland
}

\begin{abstract}
We introduce an inhomogeneous irregular-particle model for reproducing the typical light-scattering features of cometary dust such as the negative polarization near the backscattering direction, and the weak increase of the backscattering intensity. The model is based on the hierarchical Voronoi-partitioning and the algorithm provides fast generation of irregular particles with a flexible control of inhomogeneity. The input parameters of the model are refractive indices, their volumetric abundances, and the number of constituents on each level. The light-scattering properties of these particles with parameters relevant to cometary dust are solved by the volume-integral-equation method. The light-scattering features of inhomogeneous particles are compared with the mixtures of homogeneous particles, and particles with the refractive index obtained by the effective-medium approximation. We show that with the inhomogeneity size of order $0.2 \mu \mathrm{m}$, the different models produce qualitatively similar scattering features while some quantitative differences are observed which have an effect on the retrieved material composition of dust.
\end{abstract}

Keywords: Cometary dust, inhomogeneity, light scattering, numerical modeling, volume-integral-equation method

\section{Introduction}

The degree of linear polarization is one of the key lightscattering parameters in the application of scattering by cometary dust, since it does not depend on the distances of the observer, the target (comet), and the source (the Sun). This means that polarimetric observations made at different epochs are comparable regardless of the observation geometry varying over time. The degree of linear polarization is defined as $P=\left(I_{\perp}-I_{\|}\right) /\left(I_{\perp}+I_{\|}\right)$, where $I_{\perp}$ and $I_{\|}$are the intensities of the electric field components $\boldsymbol{I}_{k}=<\boldsymbol{E}_{k} \cdot \boldsymbol{E}_{k}^{*}>$ perpendicular and parallel to the scattering plane, respectively (the plane spanned by the Sun, the observer, and the comet). The phase angle $\alpha$ is the angle between the incident light onto the comet and the observed light scattered from the comet in the scattering plane.

Electromagnetic scattering by cometary dust particles is known to have some common characteristics such as a weak enhancement of the intensity and a bell-shaped polarization curve with a negative branch near the backscattering direction and the maximum positive polarization near 90 degrees at visible wavelengths. These scattering features have been observed in many different comets regardless of the size or activity of the particular comet. Nevertheless, the deepness of the negative polarization branch and the maximum positive polarization can vary between different comets, and are wavelength dependent (Kolokolova et al., 2004). In addition, the degree of linear polarization may have spatial and temporal variations. Light-scattering properties of dust depend on the size, shape, composition, and density of the dust particles. Since the dis-

\footnotetext{
${ }^{*}$ Corresponding author. Tel.: +358504160605 . E-mail address: johannes.markkanen@helsinki.fi
}

tances between individual dust particles in the coma are sufficiently large and comae are typically optically thin, scattering by a cloud of dust can be treated as a single-scattering problem, i.e., the electromagnetic coupling between particles can be neglected. Hence, finding a single particle model that reproduces the light-scattering observations, may reveal some important information of the physical composition of comets. However, it is important to keep in mind that the inverse problem of light scattering is ill-posed, and therefore, some restrictions are required for the size, composition, and structure of dust particles to have physically meaningful results (Lumme, 2000).

Infrared spectroscopy, in situ observations, and analyses of interplanetary dust particles (IDPs) collected from the Earth's stratosphere have revealed that cometary dust particles are porous heterogeneous mixtures of rock-forming elements such as $\mathrm{Mg}, \mathrm{Si}, \mathrm{Ca}, \mathrm{Fe}$, and carbonaceous materials, the so-called CHON-component consisting of light elements: carbon, hydrogen, oxygen, and nitrogen (Hanner and Bradley, 2004). The rock-forming and carbonaceous components are thought to be mixed at sub-micrometer scales (Lawler and Brownlee, 1992). Dust particles may contain micrometer-sized mineral grains coated or glued together by carbonaceous material as suggested by the microscopic images of IDPs (Bradley, 2004; Keller et al., 2000) as well as the dust formation model by Greenberg ( $\mathrm{Li}$ and Greenberg, 1997; Greenberg and Hage, 1990; Greenberg, 1982).

In light-scattering simulations, cometary dust particles are most often modelled as homogeneous aggregated structures (Lumme and Penttilä, 2011; Zubko et al., 2011; Shen et al., 2009; Kimura et al., 2006; Petrova et al., 2000; Xing and Hanner, 1997; Mukai et al., 1991), in which the refractive index is obtained using the effective-medium approximation or left as 
a free parameter. Homogenization of complex heterogeneous media with intrinsic microstructure is a sufficient approximation if the inclusions are uniformly distributed and small enough compared to the exciting wavelength. At visible wavelengths, however, the internal composition of cometary dust particles may contain inclusions with sizes comparable to the wavelength. This suggests that these inclusions should be modelled explicitly with an inhomogeneous particle.

There exists only a few models, in which inhomogeneity is explicitly accounted for. In (Yanamandra-Fisher and Hanner, 1999), the discrete-dipole approximation (DDA) was applied to study scattering by small inhomogeneous aggregates. They, however, considered only small-scale inhomogeneities changing the refractive index in randomly chosen DDA cells. Larger spheroidal inclusions in the host spheroid were considered in (Gupta et al., 2006). Both of these attempts failed to reproduce the negative-polarization branch at small phase angles.

An inhomogeneous dust model, based on the core-mantle structure, was studied in (Lasue et al., 2009; Levasseur-Regourd et al., 2007; Lasue and Levasseur-Regourd, 2006). This model involves submicrometer-sized silicate cores covered by organic refractory mantles as constituents of the ballistic particlecluster (BPCA) and ballistic cluster-cluster (BCCA) aggregates, being consistent with the dust-grain formation model ( $\mathrm{Li}$ and Greenberg, 1997). They concluded that the scattering properties of these grains are similar to those of the pure organic grains assuming that the organic mantles are thick enough. Hence, they used pure organic grains to mimic core-mantle particles. Effects of coating layers were studied in (Lindqvist et al., 2009), and they found significant differences in the polarization, but they considered only weakly absorbing particles.

In this paper, we study numerically the influence of inhomogeneity on the light scattering by cometary dust particles. The inhomogeneous particle model is compared with the effectivemedium approximation using the Maxwell-Garnett mixing rule as well as mixtures of different homogeneous particles. We use the volume-integral-equation solver in light-scattering computations introduced in section 2.1, and an irregular inhomogeneous particle model, based on the Voronoi partitioning (also known as Dirichlet tessellation) (Voronoi, 1908; Dirichlet, 1850), described in section 2.2. Section 3 presents the simulation results, and section 4 contains discussion and conclusions.

\section{Methods and models}

In this section, we introduce the numerical procedure used for solving light scattering by irregularly shaped particles, and describe the inhomogeneous irregular particle model for the analysis of light scattering by cometary dust.

\subsection{Volume-integral-equation solver}

Consider a dielectric object $\mu=\mu_{0}$ bounded by a volume $\Omega$ in a homogeneous background with permittivity $\epsilon_{0}$. The relative permittivity function $\epsilon_{r}(\boldsymbol{r})$ (the refractive index is defined as $m=\epsilon_{r}^{2}$ when $\mu=\mu_{0}$ ) is a function of position $\boldsymbol{r}$. We write a volume-integral-equation formulation for the equivalent electric volumetric (polarization) current $\boldsymbol{J}=-i \omega \epsilon_{0}\left(\epsilon_{r}-1\right) \boldsymbol{E}$ as

$$
\boldsymbol{J}^{i n c}=\boldsymbol{J}-\left(\overline{\bar{\epsilon}}_{r}-\overline{\bar{I}}\right) \cdot\left(\nabla \nabla \cdot+k_{0}^{2}\right) \int_{\Omega} G_{0} \boldsymbol{J} \mathrm{d} V
$$

where $G_{0}$ is the Green's function of the background, $k_{o}$ is the wave number in the background, and $\boldsymbol{J}^{i n c}=-i \omega \epsilon_{0}\left(\epsilon_{r}-1\right) \boldsymbol{E}^{i n c}$ is the incident current due to the incident electric field $\boldsymbol{E}^{i n c}$.

The volume of the scatterer is divided into tetrahedral elements $T_{k}$, and the unknown function $\boldsymbol{J}$ is expanded with piecewise constant basis functions $\boldsymbol{b}_{k}^{i}$ as

$$
\boldsymbol{J} \approx \sum_{k, i} c_{k}^{i} \boldsymbol{b}_{k}^{i}=\sum_{k=1}^{N_{\text {tet }}} \Pi_{k}\left(c_{k}^{x} \hat{\boldsymbol{e}}_{x}+c_{k}^{y} \hat{\boldsymbol{e}}_{y}+c_{k}^{z} \hat{\boldsymbol{e}}_{z}\right) / \sqrt{V_{k}},
$$

where $c_{k}^{i}$ are the unknown coefficient, $\hat{\boldsymbol{e}}_{x, y, z}$ are the basis vectors in the Cartesian coordinate system, and $V_{k}$ is the volume of the tetrahedron $T_{k} . \Pi_{k}$ is the step function, i.e., its value is one inside $T_{k}$ and zero otherwise. The continuous integral equation is converted into a system of linear equations by Galerkin's technique, i.e., testing $\boldsymbol{t}_{m}^{i}$ and basis functions $\boldsymbol{b}_{n}^{j}$ are identical. To reduce the singularity of the kernel, the derivatives are moved into the testing and basis functions. And using the divergence theorem, the expression for the elements of the system matrix reads as

$$
\begin{gathered}
A_{m n}^{i j}=\int_{T_{m}} \boldsymbol{t}_{m}^{i} \cdot \boldsymbol{b}_{m}^{j} \mathrm{~d} V \\
+\int_{\partial T_{m}} \boldsymbol{n}_{m} \cdot\left(\overline{\bar{\tau}}^{T} \cdot \boldsymbol{t}_{m}^{i}\right) \cdot \int_{\partial T_{n}} G_{0} \boldsymbol{n}_{n}^{\prime} \cdot \boldsymbol{b}_{n}^{j} \mathrm{~d} S^{\prime} \mathrm{d} S \\
-\int_{T_{m}} \boldsymbol{t}_{m}^{i} \cdot \overline{\bar{\tau}} \cdot k_{0}^{2} \overline{\bar{I}} \cdot \int_{T_{n}} G_{0} \boldsymbol{b}_{n}^{j} \mathrm{~d} V^{\prime} \mathrm{d} V
\end{gathered}
$$

in which $\overline{\bar{\tau}}=\left(\epsilon_{r}-1\right) \overline{\bar{I}}, \boldsymbol{n}_{m}$ is the unit normal vector of the element $T_{m}$, and $\partial T_{m}$ is a closed surface of the tetrahedron $T_{m}$. The integrals are computed by the Gauss-Legendre quadrature in combination with the singularity extraction method Järvenpää et al. (2003). The elements of the force vector read as

$$
f_{m}^{i}=\int_{T_{m}} \boldsymbol{t}_{m}^{i} \cdot \boldsymbol{J}^{i n c} \mathrm{~d} V .
$$

More details of the method can be found in (Markkanen et al., 2012). The resulting system is solved iteratively by the GMRES method, in which the matrix-vector multiplications are accelerated by the precorrected-FFT algorithm (Phillips and White, 1997). The main advantage of this formulation over the widely used DDA method is that the testing functions span the proper finite-dimensional vector space, hence, the boundary conditions across material interfaces are well-tested.

The method has computational complexity of $O\left(N_{i t e r} N \log N\right)$ for time and $O(N)$ for memory where $N$ is the number of unknowns and $N_{\text {iter }}$ is the number of iterations required to solve the system. $N_{\text {iter }}$ is generally much smaller than $N$ and it depends on the refractive index, size, and shape of the particle (Markkanen, 2014). The asymptotical complexity of this method is, therefore, the same as in the DDA. 


\subsection{Particle model}

We model cometary dust as irregularly shaped inhomogeneous particles consisting of carbonaceous materials and silicate minerals which are generally considered to be the most abundant components in comets (see, e.g., (Hanner and Bradley, 2004) and references therein). Silicates can be associated with weakly absorbing materials with the refractive index between $m=1.5+0 i$ and $1.7+0.01 i$ at wavelengths of visible light, and in this work, we assume silicate minerals to consist of $\mathrm{Mg}$-rich pyroxene with the constant refractive index $m=1.63+0.003 i$ (Dorschner et al., 1995). Carbonaceous components are associated with highly absorbing materials of $m=2.0+0.2 i$ (Jenniskens, 1993), (Li and Greenberg, 1997).

The shape of the irregular particle is generated in a manner similar to the agglomerated debris particles (see, e.g., (Zubko et al., 2009)). The algorithm starts by discretizing a sphere by tetrahedral elements. Our numerical solver uses tetrahedral mesh to describe the particle shape, but the same algorithm applies also for cubic elements used, e.g., in many DDA or FDTD (finite-difference time-domain) codes. After meshing, $N_{1}$ random seed points are generated inside a box enclosing the discretized sphere, and each seed point is associated with a subdomain index. The next step is to find corresponding Voronoi-cells for each seed. A Voronoi-cell, associated with the seed point, consists of all tetrahedral elements whose centers are closer to the corresponding seed than any other seed point (Aurenhammer and Klein, 2000). The result is a Voronoipartitioned sphere shown in the left-hand side of Fig. 1. The large-scale irregular structure is obtained by removing all the Voronoi-cells touching the surface of the sphere, and randomly removing inner cells with probability $P_{1}^{r m}$. On the right in Fig. 1, 50 percent of the inner cells have been removed, i.e., $P_{1}^{r m}=0.5$.

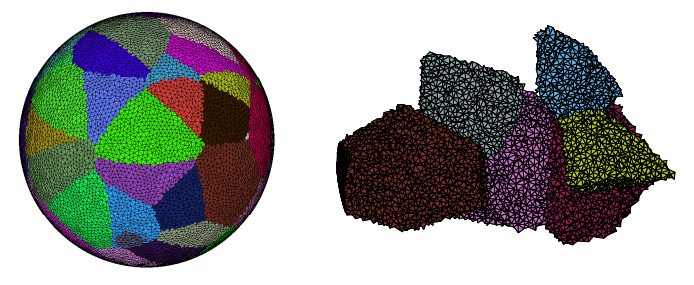

Figure 1: Generation of a level-1 Voronoi particle.

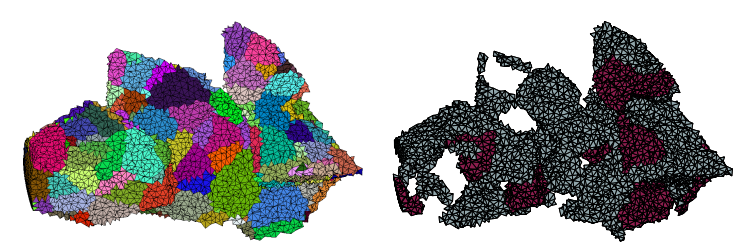

Figure 2: Generation of an irregular inhomogeneous particle from the level-1 Voronoi particle.

The resulting particle is used as an initial shape of the level2 Voronoi-particle. By choosing $N_{2}$ random seed points and finding the corresponding Voronoi-cells, the level-2 Voronoiparticle is obtained (Fig. 2, left). Porosity in the level-2 is introduced by removing cells randomly with the porosity parameter $P_{2}^{r m}$. The inhomogeneity is imposed by randomly choosing the material parameter $\epsilon_{i}$ with the probability $P_{2}^{\epsilon_{i}}$ for each Voronoi-cell on level-2 as illustrated in Fig. 2. The particle generation is a random process, hence, individual particles generated with the same parameters differ from each other but, statistically, they are the same type of particles. Figs. 3 and 4 illustrate histograms of the packing density and material abundances in a set of generated particles, respectively. One hundred particles are generated with parameters $N_{1}=100, N_{2}=1000$, $P_{1}^{r m}=P_{2}^{r m}=0.5, P_{2}^{\epsilon_{1}}=0.7$, and $P_{2}^{\epsilon_{2}}=0.3$. Hence, we have generated particles with mean packing density of 0.13 containing $70 \%$ of carbonaceous materials $\left(\epsilon_{1}\right)$ and of $30 \%$ silicates $\left(\epsilon_{2}\right)$.

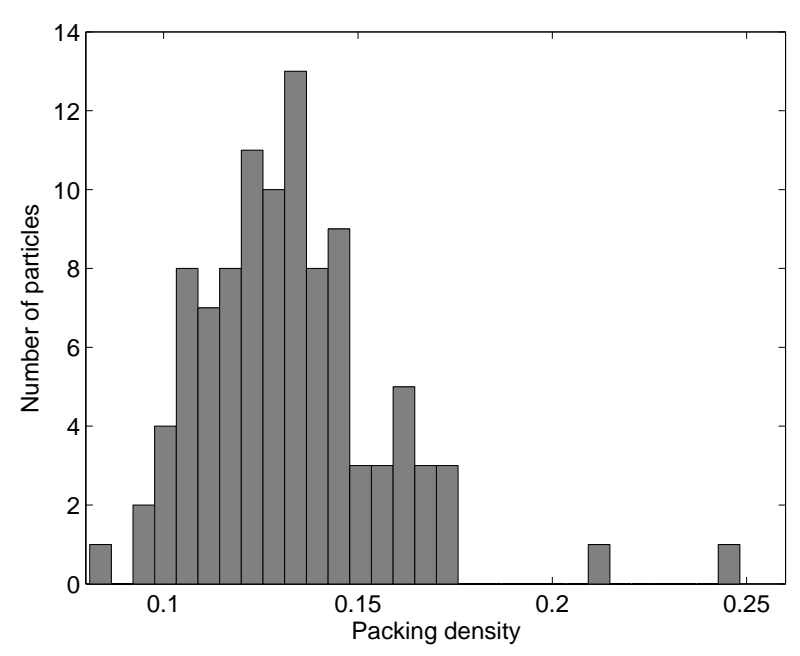

Figure 3: Packing-density distribution of generated particles.

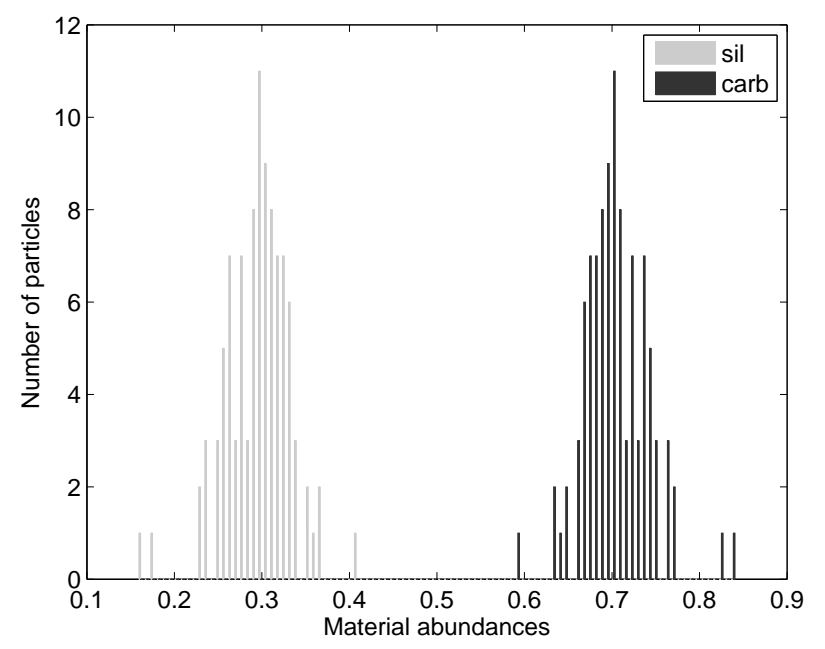

Figure 4: Material abundances in an example set of 100 generated particles aiming at a mixture of carbonaceous (70\%) and silicate material $(30 \%)$. 
It is worth noting that the generation of these particles is a relatively fast process in comparison to the solution time. This becomes an important factor when averaging over particle shapes is required as it is the case in the application to cometary dust. We want to average over particles rather than over orientations of a single particle since the real dust particles are not identical. The BCCA or BPCA aggregation processes may be timeconsuming when the constituents grains are irregular, hence, averaging over particle realizations may take too much computational time.

\section{Results}

In this section, we study the scattering properties of irregular inhomogeneous particles and compare the results with the effective-medium approximation and mixtures of homogeneous particles. All computations have been carried out by the CSC supercluster Taito using the MPI-parallelized version of the volume-integral-equation solver presented in Section 2.1. One computing node was used in computations equipped with two eight-core Intel Xeon 2.6GHz processors having $4 \mathrm{~GB}$ of memory per core. With our code, computing the scattering matrix for one particle takes from few seconds to 5 minutes depending on the size and the refractive index of the particle. Memory requirements vary from few tens to few hundreds $\mathrm{MB} /$ core. The generation of a particle takes less than a second without parallelization, hence it is negligible as compared to the total simulation time.

Particle shapes are generated by the algorithm described in Section 2 with the parameters $N_{1}=100, N_{2}$ is chosen such that the average grain size $a_{g}=0.2 \mu \mathrm{m}$, i.e., $N_{2}=\left\lceil\left(a / a_{g}\right)^{3}\right\rceil$. For example, $N_{2}=1$ for the particle size $a=0.2 \mu \mathrm{m}$, and $N_{2}=1000$ for the particle size $a=2.0 \mu \mathrm{m}$. Voids are introduced with parameters $P_{1}^{r m}=P_{2}^{r m}=0.5$, and material abundances with varying parameters $P_{2}^{\epsilon_{1}}$ and $P_{2}^{\epsilon_{2}}$ of two different materials, carbonaceous and silicate. Inhomogeneity is, therefore, imposed in the level-two with the average grain size (inhomogeneity scale) of $0.2 \mu \mathrm{m}$. This is also the average size of voids. We emphasize that the material parameters used in this study cannot be directly associated with the refractive index of real dust. We assume that the dust is composed of two materials; weakly absorbing with $m=1.63+0.003 i$ and highly absorbing with $m=2.0+0.2 i$. Both indices are assumed to be independent of the wavelength. For the silicate-type material, this may be a realistic assumption (Dorschner et al., 1995), but some carbonaceous materials, e.g., amorphous carbon, organic refractories, etc., are known to be strongly dependent on the wavelength in the visible (Jenniskens, 1993). Moreover, the nature of the carbonaceous component in comets is still unclear. It may contain complex organic molecules, organic refractory materials, amorphous carbon, etc., hence we use the effective value $m=2.0+0.2 i$ to mimic the carbonaceous component.

Polarization phase curves for different inhomogeneities and different particle sizes are presented in Fig. 5, and the corresponding phase functions $\left(S_{11}\right.$ element of the Mueller matrix) normalized to the geometric albedo at phase angle $\alpha=0$
(Hanner et al., 1981) in Fig. 6. The size of the particle is described by the volume-equivalent size parameter $k a$, where $k$ is the wave number, and $a$ is the radius of the equal-volume sphere. The average grain size parameter is $k a=0.9$. For each size, the scattering matrix is averaged over 200 particle realizations and each particle is averaged over 64 scattering planes. Each particle realization, created with the same parameters $N_{1}, N_{2}, P_{1}^{r m}, P_{2}^{r m}, P_{2}^{\epsilon_{1}}, P_{2}^{\epsilon_{2}}$, has different random shape and random distribution of inhomogeneities. Therefore, averaging over particle realization gives more reliable results than averaging over orientations of a few particles.

Typical scattering characteristics observed in comets can be described as follows. A bell-shaped polarization curve with the maximum (0.1-0.3) at $90-100^{\circ}$, and the negative branch of polarization at small phase angles $\alpha<25^{\circ}$ with the minimum of -0.02 (Kolokolova et al., 2004). The geometric albedo is usually low $A_{p} \approx 0.05$ having neutral or slightly red color (Kolokolova et al., 2004). The intensity phase function shows a strong forward scattering $\left(\alpha=180^{\circ}\right)$ peak, and a weak backscattering $\left(\alpha=0^{\circ}\right.$ ) peak with the minimum around $\alpha=55^{\circ}$. The difference in the intensities between forward and backscattering direction is around 3 decades (Schleicher, 2010).

In Figs. 5 and 6 we can see that:

1. Pure silicate particles (Carb./Sil. $=0.0$ ) have too deep negative polarization. The maximum polarization is too low for larger particles, and the location of maximum is at $\alpha<90^{\circ}$. Moreover, the albedo is too high for particles larger than $k a>3$. The shapes of the intensity curves are also unrealistic, namely, the minimum is not around $\alpha=55^{\circ}$.

2. In the case of Carb./Sil. $=0.3$, the maximum polarization is slightly higher for larger particles compared to pure silicates but the maximum is still at $\alpha<90^{\circ}$. Also the albedo is too high, and the shape is unrealistic.

3. Carb./Sil. $=0.5$ composition shows a realistic looking polarization curves except for small particles. For larger particles, position of the maximum polarization is shifted towards more realistic phase angles $\alpha=90-100^{\circ}$. Furthermore, this shows a good albedo data, especially for larger size parameters.

4. Carb./Sil. $=0.7$ case shows clearly the shifting of the maximum polarization towards larger phase angles. The negative polarization is weakened compared to the cases with more silicates. Although, it reached the observed value of -0.02 when particles are large enough. The intensity data seem to be realistic for larger particles.

Thus, the location of maximum polarization, low geometric albedo, and the intensity phase function indicate that cometary dust should consist of rather large particles, and contain absorbing materials while the negative polarization suggest the abundance of weakly absorbing components. Therefore, a mixture of highly and weakly absorbing materials may be a good candidate for explaining the scattering features of cometary dust. Based on this model, we may speculate that cometary dust contain around 50-70 \% of absorbing material. However, we emphasize that different model parameters, e.g., grain size and 

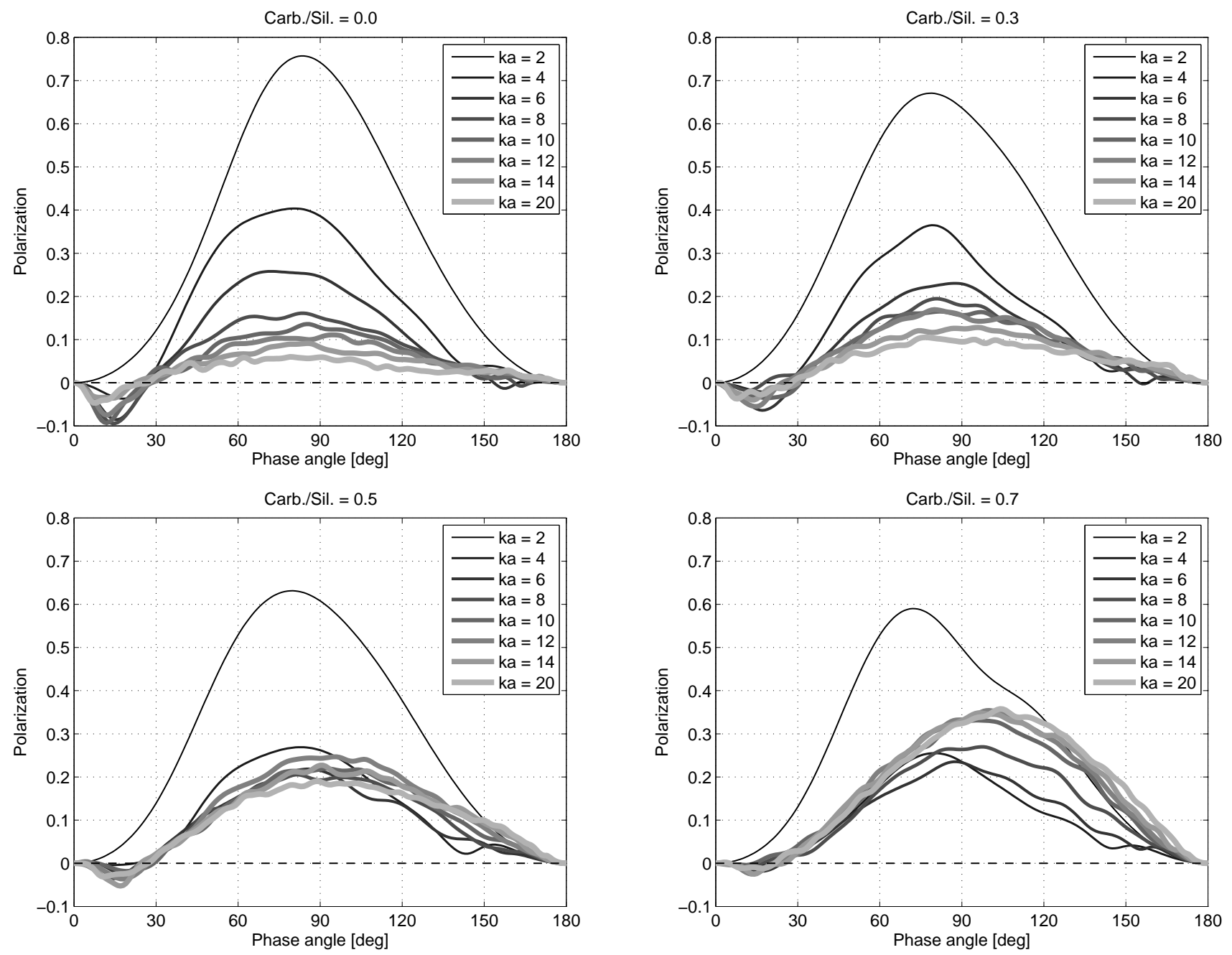

Figure 5: The degree of linear polarization for the sub-micrometer-scale mixture of carbonaceous $(m=2.0+0.2 i)$ and silicate $(m=1.63+0.003 i)$ materials. The average size parameter of the inclusions is constant at $k a=0.9$, whereas the size of the particle varies between $2 \leq k a \leq 20$.

porosity, may affect the results. Moreover, red color suggests wavelength-dependent refractive indices, namely, the imaginary part should decrease with increasing wavelength.

Next, we average the results over the differential power-law size distribution $\left(a^{-2.0}\right)$, and compare the results to the effectivemedium theory model, and the mixture of homogeneous particle model. The lower cut-off size is set to $a=0.2177 \mu \mathrm{m}$ corresponding the size parameter $k a=2$ at wavelength $684 \mathrm{~nm}$, and the upper cut-off $a=2.177 \mu \mathrm{m}(k a=20 @ \lambda=684 \mathrm{~nm})$. Due to the porosity of the particle, the radius of the circumscribing sphere is approximately twice the length of $a$.

Fig. 7 shows the degree of linear polarization and Fig. 8 the intensity normalized to the geometric albedo as a function of phase angle calculated for five different carbon/silicate mixtures averaged over the abovementioned size distribution. The labels indicate the amount of carbonaceous component in the mixture, e.g., $0 \%$ corresponds to pure silicate particles and $100 \%$ indicates pure carbonaceous particles. Three types of mixtures are presented. The first row shows the inhomogeneous particle model (internal mixture). On the second row, a mixture of ho- mogeneous particles is presented (external mixture). The third row illustrates the effective medium approximation where the refractive index is obtained by the Maxwell-Garnett mixing formula. We note that mixtures of homogeneous agglomerated debris particles have been used earlier in the analysis of cometary dust in (Zubko et al., 2014), and the effective-medium approximation is commonly applied, e.g., in (Kimura et al., 2006). Therefore, it is interesting to compare these different models. As for illustration, the polarimetric observations of the comet C/1995 O1 Hale-Bopp are also plotted. The center wavelength of the observations shown on the left column is $365 \mathrm{~nm}$ with a passband of $80 \mathrm{~nm}$, and $684 \mathrm{~nm}$ with a $90 \mathrm{~nm}$ filter on the right column. The polarimetric data is obtained from the database (Kiselev et al., 2006).

The polarization curves in Fig. 7 indicate that the submicrometer-scale two-component (low/high absorption) inhomogeneity affects the degree of linear polarization similarly to the mixture of homogeneous particles, but some differences can be observed in comparison to the effective medium approximation. The negative polarization at small phase angles ( $\alpha=0$ - 

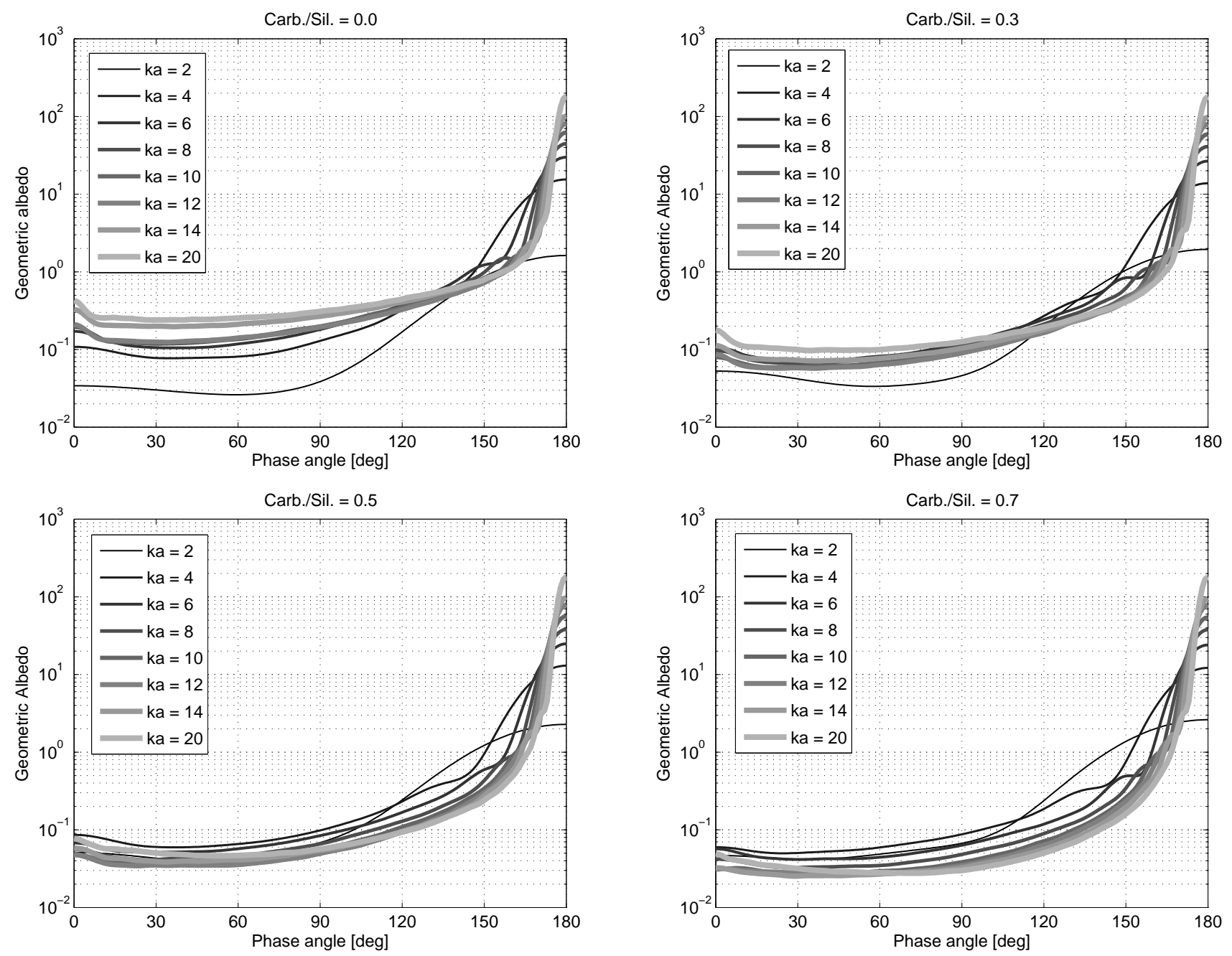

Figure 6: The phase functions normalized to the geometric albedo for the same settings as in Fig. 5

$-30^{\circ}$ ) increases as the low absorbing material becomes more abundant. The minimum shifts towards smaller phase angles in the case of the inhomogeneous model whereas it stays constant in the mixture of homogeneous particles. At phase angles of $30-60^{\circ}$, the inhomogeneity has very small effect on the polarization. The maximum polarization takes place around $90^{\circ}$, and adding more highly absorbing material into the mixture, increases the value of maximum polarization, and the location of maximum is shifted towards higher phase angles. This, however, does not hold for the effective-medium approximation when small particles are dominant (red light). Moreover, we observe some quantitative differences between these different models. In the particle mixture, highly absorbing particles seem to have much weaker influence on the total polarization and low-absorbing particles are more dominant. In the inhomogeneous mixture, the polarization curves for different compositions seem to be evenly distributed.

The computed polarization curves between the phase angles of $30-50^{\circ}$ can be conveniently fitted to the observational polarimetric data by changing the size distribution parameters, the lower cut-off and the power-law index. The higher cut-off limit is not that important as long as it is high enough since when the size distribution follows the power-law, the contribution of large particles is relatively weak, especially if absorbing materials are abundant (Zubko, 2013). It is interesting to note that the particle composition has a very weak effect on the polarization in this range. Hence, for meaningful results it is important to fit the polarimetric data in the phase angles of $\alpha<30^{\circ}$ and $\alpha>60^{\circ}$, where the inhomogeneity has a clear effect on the degree of linear polarization.

The intensity curves seem to be weakly affected by the different models. Mainly, we observe some quantitative differences as can be seen in Fig. 8. The mixture of particles leads to almost linearly behaving geometric albedos with respect to the composition while in the inhomogeneous model and the effective medium approximation, the behavior if clearly non-linear. The behavior of the geometric albedo is typical, i.e., the geometric albedo decreases as the amount absorbing material increases in a mixture. Color is blue for weakly absorbing particles, and neutral for absorbing particles. Red color observed in comets can be explained by taking the wavelength dependence of the absorbing material into account. For organic refractory mate- 

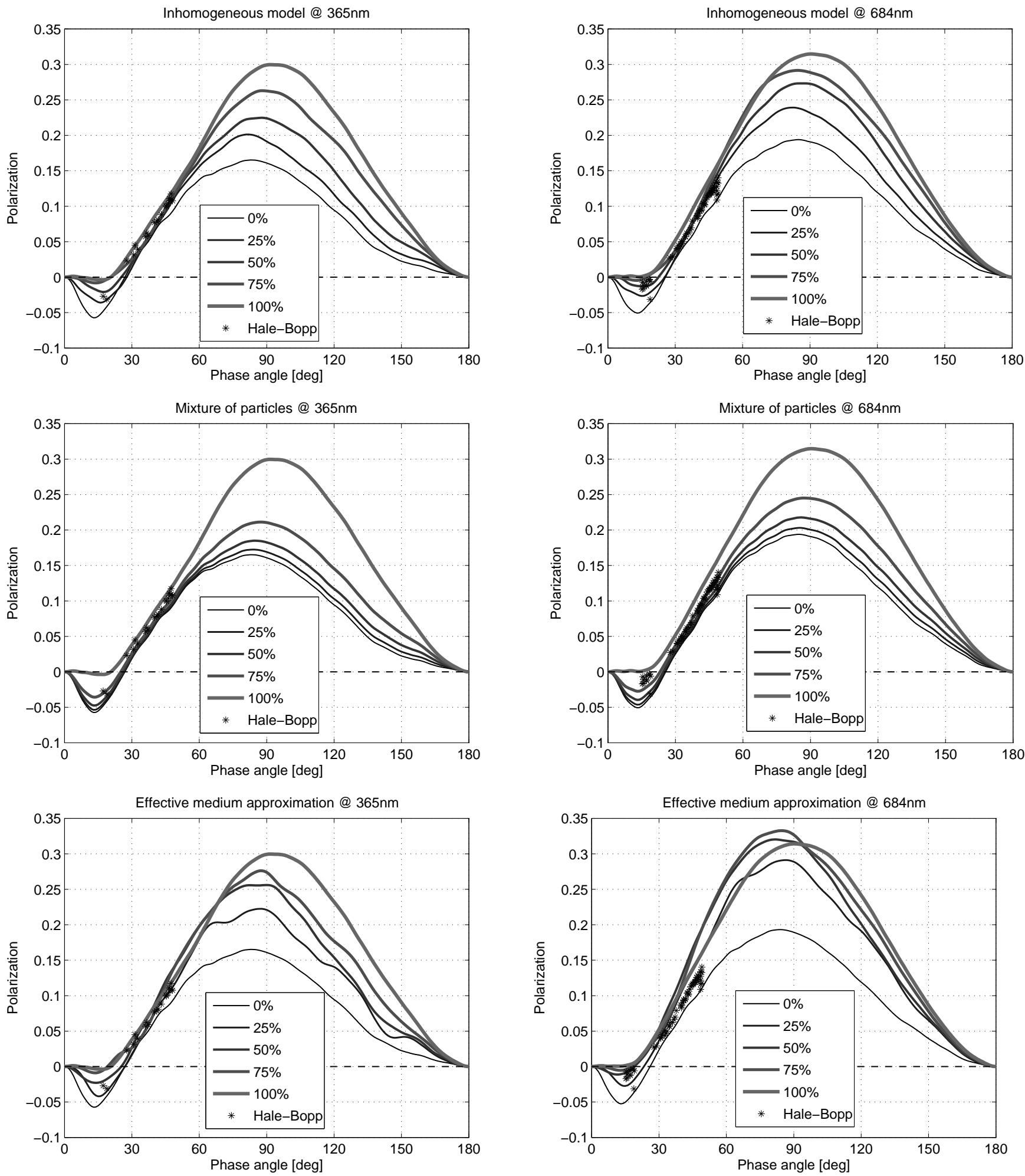

Figure 7: The degree of linear polarization for different mixtures of carbonaceous material and silicate-type mineral calculated at $\lambda=365 \mathrm{~nm}$ (left column) and $\lambda=684 \mathrm{~nm}$ (right column). Inhomogeneous model with $0.2-\mu \mathrm{m}$ scale mixture is shown on the first row. The mixture of homogeneous silicate and carbonaceous particles is on the second row. The third row illustrates the effective-medium approximation (Maxwell-Garnett).

rial, the imaginary part of the refractive index decreases from blue to red (Jenniskens, 1993) which suggests that the geometric albedo should increase in red light.

\section{Discussion and conclusions}

We have introduced an inhomogeneous irregular-particle model for the light-scattering analysis of cometary dust. This 

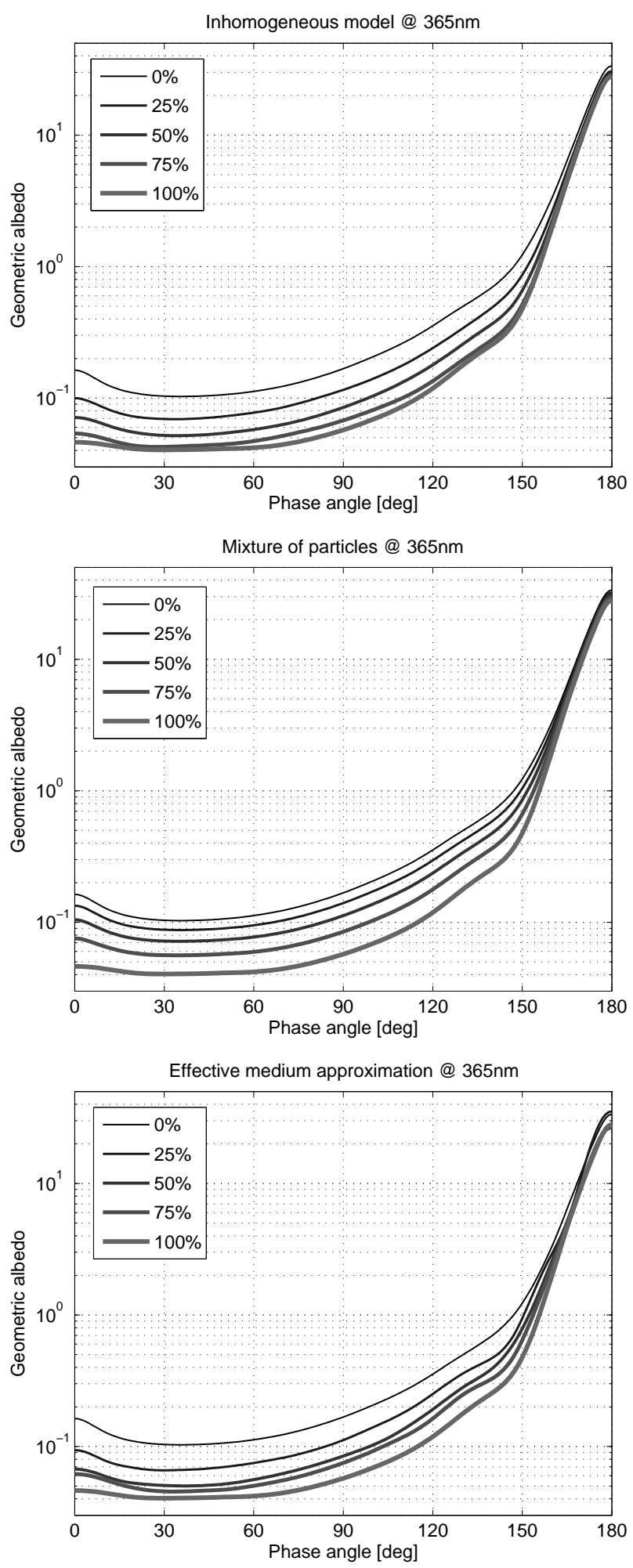
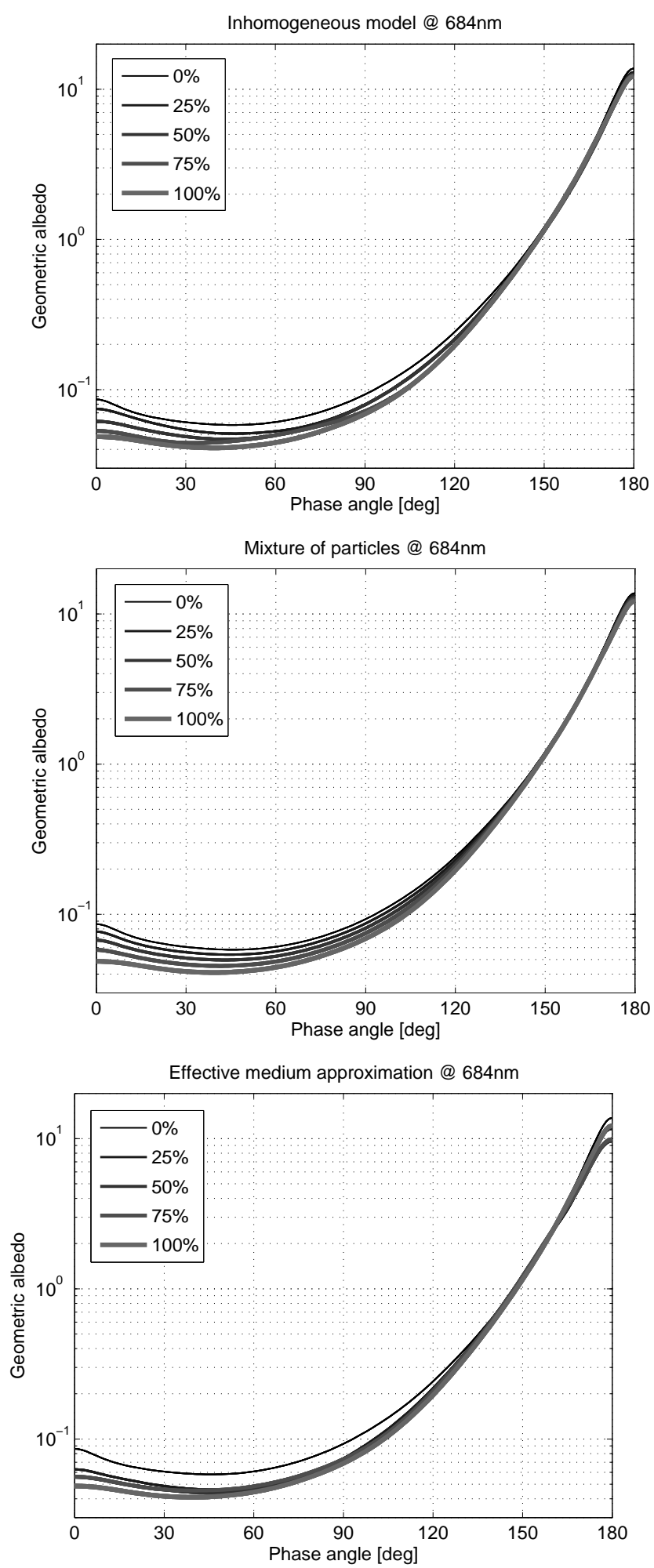

Figure 8: The phase function normalized to the geometric albedo for the same settings as in Fig. 7.

model can be useful not only for cometary but also for other types of dust, e.g., interplanetary, protoplanetary, and atmospheric dust. The model is based on the hierarchical Voronoipartitioning of the tetrahedral mesh. The particle generation algorithm provides a fast method for producing irregular inhomo- geneous particles with a flexible control of inhomogeneity. The particle model was applied to analyze scattering by cometary dust, and the results were compared with the effective-medium approximation model and the mixtures of homogeneous particles. 
Cometary dust was modelled as porous inhomogeneous irregular particles with refractive indices corresponding to carbonaceous and silicate-type materials which are assumed to be the most abundant constituents in cometary dust. Our simulation results agree with the previous studies. Pure silicatetype particles (low absorption) produce a deep negative polarization branch and low minimum of the positive polarization. Highly absorbing particles, in turn, show a weak negative polarization branch and high positive polarization with the same size distribution of particles. The location of the maximum polarization depends on the amount of absorbing particles. For weakly absorbing particles, the maximum is at $\alpha<90^{\circ}$, and, for highly absorbing particles at $\alpha>90^{\circ}$, assuming that particles are large enough. Sub-micrometer scale mixtures of these two components can be used to control the polarization characteristics, e.g., adding silicate grains into a carbonaceous host particle deepens the negative polarization branch and decreases the maximum polarization. Interestingly, compositions of particles seem to have only a weak effect on the slope of the polarization curve in the phase angles between $30-60^{\circ}$. The slope is mostly affected by the size distribution of particles. Hence, it is difficult to say anything about the internal composition of dust by looking at the polarimetric observations in this range. The internal composition has a clearer effect on the polarization at larger phase angles $\alpha>60^{\circ}$.

Material composition has also a clear effect on intensity curves. In general, the geometric albedo decreases as absorption increases (assuming the wave impedance is fixed). Our results also show that increasing the number of sub-micrometer sized absorbing grains in the silicate host, lowers the albedo. The photometric observations of comets show low albedo suggesting that absorbing materials should be major constituents in cometary dust. Our simulation results suggest that the ratio of absorbing to weakly absorbing materials is around 0.5-0.7. Moreover, the shape of the intensity function indicate that dust should consist of rather large absorbing particles. Observed red color suggests wavelength dependent material parameters. It should be noted, however, that we have used a specific refractive indices, porosity, and grain size. Therefore, these results cannot be generalized for different parameters. The inhomogeneous particle model was compared with the homogeneous particle model in which the refractive index was calculated by the Maxwell-Garnett effective-medium approximation and with the model containing a mixture of homogeneous particles. All of these models lead roughly to the same type of polarization and brightness features. However, some quantitative differences were observed which will affect the parameters retrieved from the model. As long as we do not know the particle composition, the inhomogeneous model may not be so useful compared to the homogeneous models. Nonetheless, if we have some knowledge of the internal structure of the particles, the inhomogeneous model may be useful. Especially, since inhomogeneities can be modelled without significantly increasing computational time.

Our simulation results in the studied parameter range overestimate the inversion angle, i.e., the phase angle where the degree of linear polarization changes sign, and the location of the minimum of the polarization. These values are typically the same for different comets. The inversion angle is around $\alpha=20^{\circ}$ and the minimum is around $\alpha=10^{\circ}$. Also, the maximum polarization should be shifted to the phase angles $\alpha>90^{\circ}$. Computed intensity curves slightly differ from the observed ones when averaged over size distribution. Location of minimum is around $\alpha=45^{\circ}$ instead of $\alpha=55^{\circ}$. Hence, in future work, we would like to try finding proper parameters, e.g., packing density and grain size, that reproduce the correct behavior of polarization and intensity. In addition, we will try different wavelength dependent refractive indices to obtain fits for the polarization curves of other comets. Especially, for the comets in which the polarimetric observations cover a larger range of phase angles. Finally, as the ESA Rosetta mission to the comet $67 \mathrm{P} /$ Churyumov-Gerasimenko gathers detailed information about the internal structure of cometary dust, inhomogeneous particle models may become more attractive in the future.

\section{Acknowledgment}

The research has been funded by the ERC Advanced Grant No 320773 entitled "Scattering and Absorption of Electromagnetic Waves in Particulate Media" (SAEMPL). The computations presented have been made using CSC computing resources. CSC is the Finnish IT center for science and is owned by the Ministry of Education

Aurenhammer, F., Klein, R., 2000. Voronoi diagrams. In: Sack, J., Urrutia, J. (Eds.), Handbook of computation geometry. North-Holland, Amsterdam, Netherlands, pp. 201-290.

Bradley, J., 2004. Interplanetary dust particles. In: Holland, H., Turekian, K. (Eds.), Treatise on Geochemistry. Elsevier, Amsterdam, pp. 689-711.

Dirichlet, G. L., 1850. Über die reduction der positiven quadratischen formen mit drei unbestimmten ganzen zahlen. Journal für die reine und angewandte Mathematik 40, 209-227.

Dorschner, J., Begemann, B., Henning, T., Jaeger, C., Mutschke, H., 1995. Steps toward interstellar silicate mineralogy. II. Study of $\mathrm{Mg}$-Fe-silicate glasses of variable composition. Astronomy and Astrophysics 300, 503.

Greenberg, J. M., 1982. What are comets made of? A model based on interstellar dust. In: Wilkening, L. (Ed.), Comets. University of Arizona Press, Tuscon, pp. 131-163.

Greenberg, J. M., Hage, J., 1990. From interstellar dust to comets-A unification of observational constraints. The Astrophysical Journal 361, 260-274.

Gupta, R., Vaidya, D., Bobbie, J., Chylek, P., 2006. Scattering properties and composition of cometary dust. Astrophysics and Space Science 301 (1-4), 21-31.

Hanner, M., Bradley, J., 2004. Physical properties of cometary dust from light scattering and thermal emission. In: Festou, M., Keller, U., Weave, H. (Eds.), Comets II. University of Arizona Press, Tuscon, pp. 555-564.

Hanner, M. S., Giese, R. H., Weiss, K., Zerull, R., 1981. On the definition of albedo and application to irregular particles. Astronomy and Astrophysics 140, 42-46.

Järvenpää, S., Taskinen, M., Ylä-Oijala, P., 2003. Singularity extraction technique for integral equation methods with higher order basis functions on plane triangles and tetrahedra. International journal for numerical methods in engineering 58 (8), 1149-1165.

Jenniskens, P., 1993. Optical constants of organic refractory residue. Astronomy and Astrophysics 274, 653.

Keller, L. P., Messenger, S., Bradley, J. P., 2000. Analysis of a deuterium-rich interplanetary dust particle (IDP) and implications for presolar material in IDPs. J. of Geophys. Research: Space Physics 105 (A5), 10397-10402.

Kimura, H., Kolokolova, L., Mann, I., 2006. Light scattering by cometary dust numerically simulated with aggregate particles consisting of identical spheres. Astronomy \& Astrophysics 449 (3), 1243-1254. 
Kiselev, N., Velichko, S., Jockers, K., Rosenbush, V., Kikuchi, S., E., 2006. Database of comet polarimetry. EAR-C-COMPIL-5-COMETPOLARIMETRY-V1.0. NASA Planetary Data System.

Kolokolova, L., Hanner, M., Levasseur-Regourd, A.-C., Gustafson, B., 2004. Physical properties of cometary dust from light scattering and thermal emission. In: Festou, M., Keller, U., Weave, H. (Eds.), Comets II. University of Arizona Press, Tuscon, pp. 577-604.

Lasue, J., Levasseur-Regourd, A., 2006. Porous irregular aggregates of submicron sized grains to reproduce cometary dust light scattering observations. JQSRT 100 (13), 220 - 236.

Lasue, J., Levasseur-Regourd, A., Hadamcik, E., Alcouffe, G., 2009. Cometary dust properties retrieved from polarization observations: Application to C/1995 \{O1\} HaleBopp and 1P/Halley. Icarus 199 (1), $129-144$.

Lawler, M. E., Brownlee, D. E., 1992. CHON as a component of dust from comet Halley. Nature 359 (6398), 810-812.

Levasseur-Regourd, A., Mukai, T., Lasue, J., Okada, Y., 2007. Physical properties of cometary and interplanetary dust. Planetary and Space Science 55 (9), $1010-1020$

Li, A., Greenberg, J. M., 1997. A unified model of interstellar dust. Astronomy and Astrophysics 323, 566-584.

Lindqvist, H., Muinonen, K., Timo Nousiainen, T., 2009. Light scattering by coated gaussian and aggregate particles. Journal of Quantitative Spectroscopy and Radiative Transfer 110 (1416), 1398 - 1410.

Lumme, K., 2000. Scattering properties of interplanetary dust particles. In: Mishchenko, M., Hovenier, J., Travis, L. (Eds.), Light scattering by nonspherical particles. Academic Press, San Diego, pp. 555-583.

Lumme, K., Penttilä, A., 2011. Model of light scattering by dust particles in the solar system: Applications to cometary comae and planetary regoliths. JQSRT 112 (11), 1658-1670.

Markkanen, J., 2014. Discrete Helmholtz decomposition for electric current volume integral equation formulation. IEEE Trans. Ant. and Propag., 62 (12), 6282-6289.

Markkanen, J., Ylä-Oijala, P., Sihvola, A., 2012. Discretization of volume integral equation formulations for extremely anisotropic materials. IEEE Trans. Ant. and Propag. 60 (11), 5195-5202.

Mukai, S., Mukai, T., Kikuchi, S., 1991. Scattering properties of cometary dust based on polarimetric data. In: Levasseur-Regourd, A., Hasegawa, H. (Eds.), Origin and Evolution of Interplanetary Dust. Vol. 173 of Astrophysics and Space Science Library. Springer, Netherlands, pp. 249-252.

Petrova, E. V., Jockers, K., Kiselev, N. N., 2000. Light scattering by aggregates with sizes comparable to the wavelength: An application to cometary dust. Icarus 148 (2), 526 - 536.

Phillips, J. R., White, J. K., 1997. A precorrected-fft method for electrostatic analysis of complicated 3-d structures. IEEE Trans. CAD of Integ. Circ. and Sys. 16 (10), 1059-1072.

Schleicher, D., 2010. http://asteroid.lowell.edu/comet/dustphase_ plots.html.

Shen, Y., Draine, B., Johnson, E., 2009. Modeling porous dust grains with ballistic aggregates. II. light scattering properties. The Astrophysical Journal 696 (2), 2126-2137.

Voronoi, G., 1908. Nouvelles applications des paramètres continus à la théorie des formes quadratiques. deuxième mémoire. recherches sur les parallélloèdres primitifs. Journal für die reine und angewandte Mathematik (Crelles Journal) 1908 (134), 198-287.

Xing, Z., Hanner, M. S., 1997. Light scattering by aggregate particles. Astronomy and Astrophysics 324, 805-820.

Yanamandra-Fisher, P., Hanner, M., 1999. Optical properties of nonspherical particles of size comparable to the wavelength of light: Application to comet dust. Icarus 138 (1), 107-128.

Zubko, E., 2013. Light scattering by cometary dust: Large-particle contribution. Earth, Planets and Space 65 (3), 139-148.

Zubko, E., Furusho, R., Kawabata, K., Yamamoto, T., Muinonen, K., Videen, G., 2011. Interpretation of photo-polarimetric observations of comet 17P/Holmes. JQSRT 112 (11), 1848-1863.

Zubko, E., Kimura, H., Shkuratov, Y., Muinonen, K., Yamamoto, T., Okamoto, H., Videen, G., 2009. Effect of absorption on light scattering by agglomerated debris particles. JQSRT 110 (1416), 1741 - 1749.

Zubko, E., Muinonen, K., Videen, G., Kiselev, N. N., 2014. Dust in comet c/1975 v1 (west). Monthly Notices of the Royal Astronomical Society 440 (4), 2928-2943. 